\title{
P126: Direct observation survey of practice of alcohol-based handrubbing in Fann Teaching Hospital, Dakar, Senegal
}

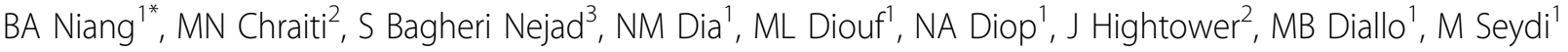 \\ From 2nd International Conference on Prevention and Infection Control (ICPIC 2013) \\ Geneva, Switzerland. 25-28 June 2013
}

\section{Introduction}

Health care-associated infections (HCAI) result essentially from cross-transmission of pathogenic microorganisms by the hands of healthcare workers (HCW). Their care represents a universal challenge in practice.

\section{Objectives}

Our study aimed to measure HCW compliance with hand hygiene.

\section{Methods}

We conducted a direct observance of hand hygiene compliance of HCW over a period of three months, based on the WHO's "five indications of the hand hygiene" approach.

\section{Results}

For a total of 338 opportunities, the rate of global observance of hand hygiene was $36.1 \%$ with $80.3 \%$ of handrubbing realized. According to the department, this rate of observance was variable: Pneumology (42.3\%), Thoracic Surgery and Cardiovascular (58.6\%), Neurology (20\%), Neurosurgery (24\%), Emergency (25\%), Laboratories (30\%), Infectious diseases (39\%), Psychiatry (33.3\%), ORL (25\%), Oral department (44.4\%). According to the professional category, the observance was the following one: doctors (50.6\%), nurses (34\%) auxiliaries (29.1\%) other nursing staffs (43.8\%). The level of use of Alcohol-based handrub (ABHR) during hand hygiene was: auxiliaries (93\%), doctors $(82.1 \%)$, nurses $(75.8 \%)$, others (14.3\%). The observance of ABHR according to "five indications" was $87.7 \%$ before patient contact, of
83.3\% before aseptic procedure, $44.4 \%$ after a risk of body fluid exposure, of $78 \%$ after patient contact and of $100 \%$ to the immediate surroundings of patient.

\section{Conclusion}

Observance of hand hygiene with ABHR is still low in the structure. A training program coupled with a sharing experience of outcomes of the survey should allow to improve it.

\section{Disclosure of interest}

None declared.

\section{Author details}

'Infectious Diseases department, FANN teaching hospital, Dakar, Senegal. ${ }^{2}$ Service of control and prevention, HUG, Geneva, Switzerland. ${ }^{3} \mathrm{WHO}$, Geneva, Switzerland.

Published: 20 June 2013

doi:10.1186/2047-2994-2-S1-P126

Cite this article as: Niang et al:: P126: Direct observation survey of practice of alcohol-based handrubbing in Fann Teaching Hospital, Dakar, Senegal. Antimicrobial Resistance and Infection Control 2013 2(Suppl 1):P126. 